\title{
Mesenchymal Deficiency of Notch1 Attenuates Bleomycin-Induced Pulmonary Fibrosis
}

\author{
Biao Hu, Zhe Wu, David Bai, Tianju Liu, Matthew R. Ullenbruch, and Sem H. Phan
}

From the Department of Pathology, University of Michigan Medical School, Ann Arbor, Michigan

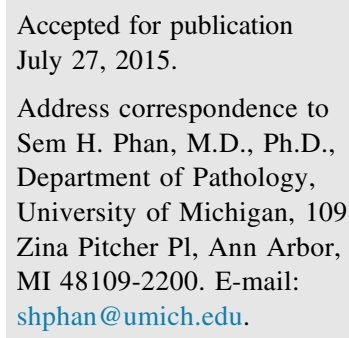

Address correspondence to Sem H. Phan, M.D., Ph.D., Department of Pathology, University of Michigan, 109 Zina Pitcher Pl, Ann Arbor, MI 48109-2200. E-mail: shphan@umich.edu.

\begin{abstract}
Notch signaling pathway is involved in the regulation of cell fate, differentiation, proliferation, and apoptosis in development and disease. Previous studies suggest the importance of Notch1 in myofibroblast differentiation in lung alveogenesis and fibrosis. However, direct in vivo evidence of Notch1-mediated myofibroblast differentiation is lacking. In this study, we examined the effects of conditional mesenchymal-specific deletion of Notch1 on pulmonary fibrosis. Crossing of mice bearing the floxed Notch1 gene with $\alpha 2(\mathrm{I})$ collagen enhancer-Cre-ER(T)-bearing mice successfully generated progeny with a conditional knockout (CKO) of Notch1 in collagen I-expressing (mesenchymal) cells on treatment with tamoxifen (Notch1 CKO). Because Notch signaling is known to be activated in the bleomycin model of pulmonary fibrosis, control and Notch1 CKO mice were analyzed for their responses to bleomycin treatment. The results showed significant attenuation of pulmonary fibrosis in CKO relative to control mice, as examined by collagen deposition, myofibroblast differentiation, and histopathology. However, there were no significant differences in inflammatory or immune cell influx between bleomycin-treated CKO and control mouse lungs. Analysis of isolated lung fibroblasts confirmed absence of Notch1 expression in cells from CKO mice, which contained fewer myofibroblasts and significantly diminished collagen I expression relative to those from control mice. These findings revealed an essential role for Notch1mediated myofibroblast differentiation in the pathogenesis of pulmonary fibrosis. (Am J Pathol 2015, 185: 3066-3075; http://dx.doi.org/10.1016/j.ajpath.2015.07.014)
\end{abstract}

Notch signaling is known to play critical roles in development, tissue homeostasis, and disease. ${ }^{1-10}$ Notch signaling is mediated via four known receptors, Notch 1, 2, 3, and 4, which serve as receptors for five membrane-bound ligands, Jagged 1 and 2 and Delta 1, 3, and 4. ${ }^{1,11-13}$ The Notch receptors differ primarily in the number of epidermal growth factor-like repeats and C-terminal sequences. ${ }^{13}$ For instance, Notch 1 contains 36 of epidermal growth factor-like repeats, is composed of approximately 40 amino acids, and is defined largely by six conserved cysteine residues that form three conserved disulfide bonds. ${ }^{1,13-15}$ These epidermal growth factor-like repeats can be modified by O-linked glycans at specific sites, which is important for their function. $1,14,15$ Modulation of Notch signaling by Fringe proteins, ${ }^{16-18}$ which are $\mathrm{N}$-acetylglucosamine transferases, illustrates the importance of these carbohydrate residues. ${ }^{16,18}$ Moreover, mutation of the GDP-4-keto-6-deoxymannose-3,5-epimerase4-reductase causes defective fucosylation of Notch1, resulting in impairment of the Notch1 signaling pathway and myofibroblast differentiation. ${ }^{19-21}$ Because myofibroblasts are important in both lung development and fibrosis, elucidation of the role of Notch signaling in their genesis in vivo will provide insight into the significance of this signaling pathway in either context.

The importance of Notch signaling in tissue fibrosis is suggested in multiple studies. ${ }^{10,21-24}$ As in other organs or tissues, pulmonary fibrosis is characterized by fibroblast proliferation and de novo emergence of myofibroblasts, which is predominantly responsible for the increased extracellular matrix production and deposition. ${ }^{25-31}$ Animal models, such as bleomycin-induced pulmonary fibrosis, are characterized by

\footnotetext{
Supported by NIH grants HL52285, HL91775, and HL112880 (S.H.P.) and utilized the National Institute of Diabetes and Digestive and Kidney Diseases grant DK020572 to Dr. John A. Williams at University of Michigan (Cell and Molecular Biology Core of the Michigan Diabetes Research and Training Center).
}

Disclosures: None declared. 
both acute and chronic inflammation with subsequent myofibroblast differentiation that mainly originated from the mesenchymal compartment. ${ }^{21,25-28}$ In vitro studies of cultured cells implicate Notch signaling in myofibroblast differentiation, ${ }^{21}$ which is mediated by induction of the Notch1 ligand Jagged1 when lung fibroblasts are treated with found in inflammatory zone $1 .^{21}$ Moreover, GDP-4-keto-6deoxymannose-3,5-epimerase-4-reductase knockout mice with defective fucosylation of Notch1 exhibit consequent impairment of Notch signaling and attenuated pulmonary fibrosis in studies using the bleomycin model. ${ }^{21}$ The in vivo importance of Notch signaling in myofibroblast differentiation during lung development has also been suggested by demonstration of impaired alveogenesis in mice deficient in lunatic fringe $^{32}$ or Notch receptors. ${ }^{10,33-35}$ These in vivo studies, however, do not pinpoint the cell type in which deficient Notch signaling is causing the observed impairment of myofibroblast differentiation. This is further complicated by the extensive evidence showing that, in addition to myofibroblast differentiation, Notch1 mediates multiple functional responses in diverse cell types, including inflammation and the immune system. ${ }^{21,36-38}$ In the case of tissue injury and fibrosis, including the bleomycin model, the associated inflammation and immune response as well as parenchymal injury can affect myofibroblast differentiation via paracrine mechanisms. ${ }^{39,40}$ Thus, although global impairment of Notch signaling can impair myofibroblast differentiation in vivo, it does not necessarily indicate a specific direct effect on the mesenchymal precursor cell. Furthermore, understanding the importance of Notch signaling in these different cell compartments is critical for future translational studies to develop effective drugs targeting this signaling pathway with minimal off-target or negative adverse effects.

In this study, the effects of conditional selective Notch1 deficiency in the mesenchymal compartment on myofibroblast differentiation and bleomycin-induced pulmonary fibrosis were examined using a Cre-Lox strategy. The transgenic Cre mice bore the $C r e-E R(T)$ gene composed of Cre recombinase and a ligand-binding domain of the estrogen receptor ${ }^{41}$ driven by a minimal promoter containing a far-upstream enhancer from the $\alpha 2$ (I) collagen gene. When activated by tamoxifen, this enhancer enabled selective Cre expression only in type I collagen-expressing (mesenchymal) cells, such as fibroblasts and other mesenchymal cells, ${ }^{42}$ leading to excision of LoxP consensus sequence flanked target gene DNA fragment (floxed gene) of interest. ${ }^{41,43-46}$ To evaluate the importance of Notch1 in the mesenchymal compartment and discriminate its effects from those in the inflammatory and immune system and other compartments, the transgenic $\operatorname{Cre}-E R(T)$ mice $\left[\operatorname{Coll} \alpha 2-\operatorname{Cre}-E R(T)^{+/ 0}\right]$ were crossed with mice harboring the floxed (containing loxP sites) Notchl gene $\left(\right.$ Notch $\left.^{f l f l}\right)$. The resulting progeny mice [Notch1 conditional knockout (CKO)] that were homozygous for the floxed Notchl allele and hemizygous for the Coll $\alpha 2-C r e-E R(T)$ allele with genotype $\left[\right.$ Notch $\left.1^{f l / l}, \operatorname{Coll} \alpha 2-\operatorname{Cre}-E R(T)^{+/ 0}\right]$ were Notch1 deficient in the mesenchymal compartment when injected with tamoxifen. Control Notch1 wild-type (WT) mice exhibited the expected pulmonary fibrosis along with induction of Jagged 1 and Notch1 on treatment with bleomycin, consistent with previous observation of Notch signaling activation in this model. ${ }^{21}$ Isolated and cultured Notch1 CKO mouse lung fibroblasts were deficient in Notch1 and exhibited diminished myofibroblast differentiation compared with cells from the corresponding WT control mice. Most important, compared with WT control mice, the CKO mice exhibited diminished bleomycin-induced pulmonary fibrosis that was accompanied by significant reduction in $\alpha$-smooth muscle actin ( $\alpha$-SMA) and type I collagen gene expression, consistent with defective myofibroblast differentiation. In contrast, enumeration of lung inflammatory and immune cells failed to show a significant difference in bleomycin-induced recruitment of these cells between control and CKO mice. Thus, selective Notch1 deficiency in mesenchymal cells caused impairment of fibrosis that is at least, in part, because of deficient myofibroblast differentiation, and without affecting the inflammatory and immune response in this animal model.

\section{Materials and Methods}

\section{Animal Treatment}

All animal protocols were approved by the University Committee on Use and Care of Animals of the University of Michigan (Ann Arbor). Mice with the first exon of Notchl gene flanked with LoxP recombination sites (Notch $\left.I^{f / f f}\right)^{47,48}$ and mice carrying a tamoxifen-inducible Cre-recombinase [Cre$E R(T)]$ under the control of a regulatory sequence from the $\alpha 2(\mathrm{I})$ collagen gene (C57BL/6J-Tg[Coll $\alpha 2-\mathrm{Cre}-E R(T)])^{46}$ were bred from breeding pairs, which were originally purchased from Jackson Laboratory (Bar Harbor, ME) and received from Dr. Benoit de Crombrugghe (University of Texas MD Anderson Cancer Center, Houston, TX), respectively. Both strains were on a C57BL/6 background. To generate mesenchymal cell-specific Notch1-deficient mice, the Notch $^{f l / f}$ mice were cross bred with Coll $\alpha 2-C r e-E R(T)^{+/ 0}$ (heterozygous allele) transgenic mice to generate mice heterozygous for both alleles. The second cross between Notch fllft mice and heterozygous mice from the first cross produced $\operatorname{Notch}^{f l / f}$, Coll $\alpha 2-C r e-E R(T)^{+/ 0}$ mice, which were used for further experiments.

For treatment of mice, a stock solution of tamoxifen (4hydroxitamoxifen; Sigma-Aldrich Co, St. Louis, MO) in $100 \mathrm{mg} / \mathrm{mL}$ ethanol was diluted in corn oil to $10 \mathrm{mg} / \mathrm{mL}$. To selectively delete Notch 1 in mesenchymal cells, adult Notch $1^{f / f l}$, Coll $\alpha 2-C r e-E R(T)^{+/ 0}$ mice (8 weeks old) and the relevant control [Coll $\alpha 2-\operatorname{Cre}-E R(T)^{+/ 0}$ with WT Notch1] mice were given daily i.p. injections of the tamoxifen suspension $(0.1 \mathrm{~mL}$ of diluted stock) for 8 days to induce mesenchymal cell-specific expression of the Cre-ER(T) recombinase and removal of the floxed Notch1 coding region accordingly. The resultant Notch1-deficient mice were referred to as Notch1 CKO mice. 
After the tamoxifen treatment regimen, the Notch1 CKO and control mice were injected endotracheally with $2 \mathrm{U} / \mathrm{kg}$ body weight of bleomycin (Blenoxane; Mead Johnson, Ramsey, NJ) dissolved in sterile phosphate-buffered saline (PBS) as before. ${ }^{44,45}$ The control group received the same volume of sterile PBS only. Daily tamoxifen treatment continued until they were sacrificed at the indicated time points after bleomycin treatment.

All animals used for experiments were genotyped by PCR to detect Notchl and $\mathrm{Cre}-E R(T)$ gene sequences, as described previously. ${ }^{45-48}$ For detection of the floxed Notchl by PCR in genotyping, the primer pairs used were as follows: $5^{\prime}$ TGGAAGCTACTGACTTAGTAGGGGGAAAAC- $3^{\prime}$ and $5^{\prime}$-GCAAGCATGAAGTGGTCCAGGGTGTGAGTG- $3^{\prime} .{ }^{48}$ For detection of the $\mathrm{Cre}-E R(T)$ gene, the following primers were used in genotyping: forward, 5'-TCCAATTTACTGACCGTACACCAA-3'; and reverse, 5'-CCTGATCCTGGCAATTTCGGCTA- $3^{\prime} .44,45$

\section{Cell Culture}

Mouse lung fibroblasts were isolated 14 days after bleomycin or saline treatment and cultured as described previously. ${ }^{44,45,49}$ Cells cultured between passages 3 and 5 after primary culture were used in experiments as indicated.

\section{Morphological Analysis}

This was performed as previously described. ${ }^{44,45}$ Briefly, 21 days after the endotracheal injection of saline or bleomycin, mice were euthanized and the lungs were thoroughly perfused with saline to remove blood from the lung vascular beds. The lungs were then removed from the thoracic cavity, inflated, and fixed in formalin, followed by paraffin embedding, dividing into sections, and staining with trichrome staining. ${ }^{44,45}$

\section{Hydroxyproline Assay}

To assess the extent of fibrosis, the hydroxyproline content of whole lung homogenates was assayed 21 days after bleomycin or saline treatment by colorimetric assay after acid hydrolysis, as described previously. ${ }^{44,45}$

\section{Western Blot Analysis}

Western blot analysis was performed essentially as before. ${ }^{44,45}$ As indicated, homogenized murine lung tissue samples or fibroblasts were lysed in Radioimmunoprecipitation Assay Protein Lysis and Extraction Buffer $(25 \mathrm{mmol} / \mathrm{L}$ Tris $\bullet \mathrm{HCl}, \mathrm{pH}$ 7.6, $150 \mathrm{mmol} / \mathrm{L} \mathrm{NaCl}, 1 \%$ Nonidet P-40, $1 \%$ sodium deoxycholate, and $0.1 \%$ sodium dodecyl sulfate) containing proteinase inhibitor cocktail (Roche Diagnostics, Mannheim, Germany). After determination of protein concentration by BCA assay (Fisher Scientific, Pittsburgh, PA), equal amounts of total protein were electrophoresed through $12 \%$ SDS-polyacrylamide gels. The separated protein bands were transferred onto Hybond P membranes (GE Healthcare Life Sciences, Marlborough, MA) and blotted against different antibodies, as indicated. The anti- $\alpha$-SMA and anti-procollagen I antibodies were purchased from Sigma-Aldrich Co, and anti-Notch1 antibody was purchased from Santa Cruz Biotechnology Inc. (Santa Cruz, CA). Equal loading was confirmed by reblotting the membrane with horseradish peroxidase-conjugated anti-glyceraldehyde-3-phosphate dehydrogenase antibody from Abcam Inc. (Cambridge, MA). The blots were scanned and the band intensities were quantified using Molecular Imaging software version 5.0.2.30 (Carestream Health, Inc., Rochester, NY). The relative intensity of target proteins was expressed after normalization to the intensity of glyceraldehyde-3phosphate dehydrogenase bands.

\section{mRNA Analysis}

Determination of mRNA for the indicated genes was undertaken using real-time PCR as before. ${ }^{44,45}$ Results were expressed as $2^{-\Delta \Delta C_{T}}$ using $18 \mathrm{~S}$ rRNA as the reference and the indicated group as calibrator. ${ }^{50}$ Total RNA was extracted from either murine lung tissue or fibroblasts, as indicated 14 days after bleomycin or saline treatment. For each assay, $200 \mathrm{ng}$ of total RNA was used as template and 18S rRNA was used as internal control to normalize the amount of input RNA. Onestep real-time RT-PCR $\left(48^{\circ} \mathrm{C}\right.$ for 30 minutes, $95^{\circ} \mathrm{C}$ for 10 seconds, followed by 50 cycles of $95^{\circ} \mathrm{C}$ for 10 seconds and $60^{\circ} \mathrm{C}$ for 1 minute) was undertaken with TaqMan One Step RT-PCR Master Mix (Applied Biosystems, Foster City, CA) using a GeneAmp 7500 Sequence Detection System (Applied Biosystems Inc., Foster City, CA). Primers and probes were purchased from Applied Biosystems.

\section{Flow Cytometry Analysis}

Flow cytometry analysis was performed as before..$^{44,45}$ Briefly, whole lung single-cell suspensions were prepared 14 days after bleomycin or saline treatment by digesting the indicated lung samples with collagenase and DNAase digestion mixture $[5 \mathrm{mg}$ collagenase and $1 \mathrm{mg}$ DNase (Worthington Biochemical Co, Lakewood, NJ) in $20 \mathrm{~mL}$ Hanks' balanced salt solution supplemented with $1 \mathrm{~mL}$ plasma-derived serum] for 1.5 hours at $37^{\circ} \mathrm{C}$. They were then sequentially filtered through $100-$ and $40-\mu \mathrm{m}$ mesh cloth, and then washed with $1 \times$ PBS. The cell suspensions were then fixed with $4 \%$ paraformaldehyde in $1 \times$ PBS and stained with fluorescent-labeled specific primary antibody of interest or corresponding IgG control in $1 \times$ PBS only or $1 \times$ PBS containing $0.1 \%$ saponin (for staining of $\alpha$-SMA) for 1 hour at room temperature. After stringent washing, they were analyzed on a Becton-Dickinson LSR-II machine (BD Biosciences, Chicago, IL). Data collected were then analyzed using Flowjo software version 7.6.5 (Tree Star Inc., Ashland, OR). For evaluation of inflammatory/immune 
A

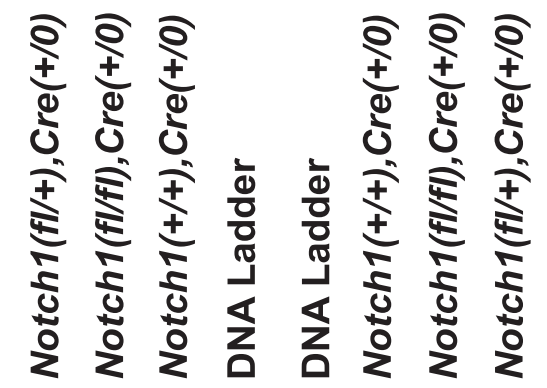

Figure 1 A: Mouse genotype analysis. Genomic DNA samples from the indicated strains of mice were analyzed using primers specific for wild-type (wt) and floxed (fl) Notch1 or Cre-ER(T) in PCRs. The PCR products and 100-bp DNA ladder were separated by agarose gel electrophoresis. The bands corresponding to the wt (317 bp) and fl (367 bp) Notch1 alleles are indicated, and confirm the correct Notch1 genotype for each strain. The correct genotype with respect to $C r e-E R(T)$ (500 bp) gene is confirmed in the respective strain. B: Lung fibroblasts were isolated from control (WT) or Notch1 conditional knockout (CKO) mice after injection of tamoxifen for 8 days. Notch1 expression was evaluated in these cells by Western blot analysis. Glyceraldehyde-3-phosphate dehydrogenase (GAPDH) is shown as loading control.

response, the whole lung cell suspension was stained with fluorescent-labeled antibodies against CD3, CD4, CD8, B220, $\mathrm{F} 4 / 80$, and $\mathrm{Gr}-1$ for enumeration of $\mathrm{T}$ cells, $\mathrm{CD} 4^{+}$and $\mathrm{CD} 8^{+} \mathrm{T}$ cells, B cells, macrophages, and neutrophils, respectively.

\section{Immunostaining}

Mouse lung fibroblasts were isolated from either Notch1 CKO mice or WT mice and cultured on cover slides overnight. The cells were then fixed with $4 \%$ formaldehyde made with $1 \times$ PBS and stained with Cy3-conjugated anti- $\alpha$-SMA antibody or Cy3-conjugated IgG control (made with $1 \times$ PBS and $0.1 \%$ saponin) for 1 hour after blocking for 1 hour with blocking buffer $(1 \times$ PBS, $1 \%$ bovine serum albumin, and $0.1 \%$ saponin). The cells were then washed extensively with washing buffer ( $1 \times$ PBS and $0.1 \%$ saponin). The slides were then viewed using a microscope, and the images were obtained at $\times 200$ magnification.

\section{Statistical Analysis}

Statistical analysis was performed as before ${ }^{44,45}$ using analysis of variance, followed, where appropriate, by post hoc testing using Scheffé's test. $P<0.05$ was used as a criterion for statistical significance.

\section{Results}

Generation of Mice with Selective Deficiency of Notch1 in the Mesenchymal Compartment

Homozygous floxed Notchl mice (Notch $1^{f / f H}$ ) with the first exon of Notchl gene flanked with LoxP recombination sites were cross bred with mice harboring a $\mathrm{Cre}-E R(T)$ recombinase

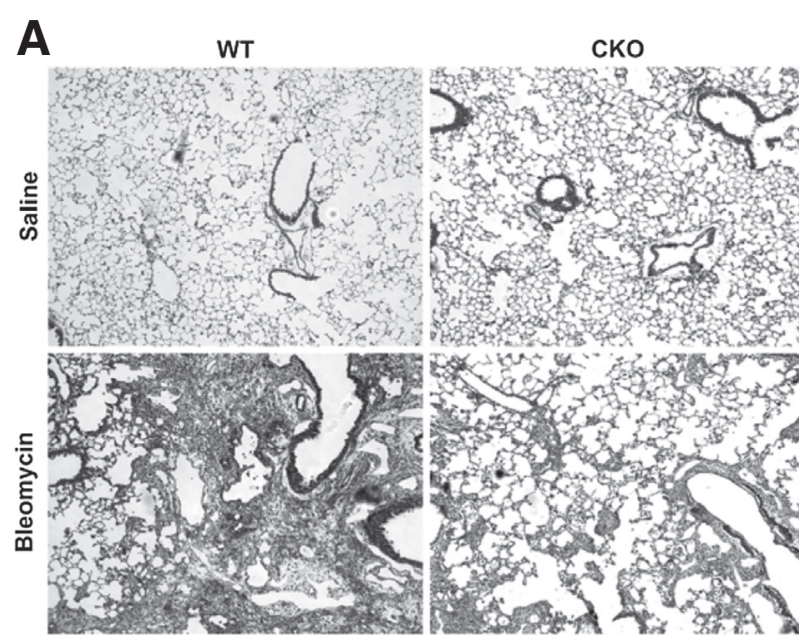

B
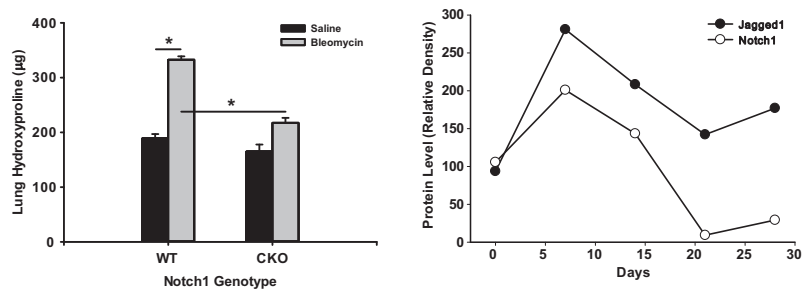

Figure 2 Effect of mesenchymal Notch1 deficiency on bleomycin-induced pulmonary fibrosis. Tamoxifen-treated control [wild-type (WT)] and Notch1 conditional knockout (CKO) mice were given endotracheal injections of either bleomycin or saline, as indicated. The lungs were harvested and analyzed for histopathology (A) and hydroxyproline content (B) 21 days later. A: Representative sections from saline- or bleomycin-treated control or Notch1 CKO mice. All were imaged at $\times 20$ final magnification. B: The lung samples were analyzed for hydroxyproline content. C: Lung tissue samples from saline (S)- or bleomycin (B)-treated WT mice at the indicated time points were analyzed for Jagged 1 and Notch1 protein by Western blot analysis. The blots were scanned and quantitated as relative integration units (relative density). A representative blot and the results of quantitation of the bands are shown. The results were expressed as a percentage of the respective saline control value at each time point. Increased expression of Jagged 1 was noted in bleomycin-treated mouse lungs at all time points examined, but only on days 7 and 14 for Notch1. Data are given as means $\pm \mathrm{SEM}(\mathbf{B}) . N=5(\mathbf{B}) .{ }^{*} P<0.05$. 
gene controlled by minimal promoter with an enhancer from the mouse $\alpha 2(\mathrm{I})$ collagen gene promoter. The progeny was then backcrossed with the Notchl $1^{f / f l}$ mice to obtain mice homozygous for the floxed Notchl allele and
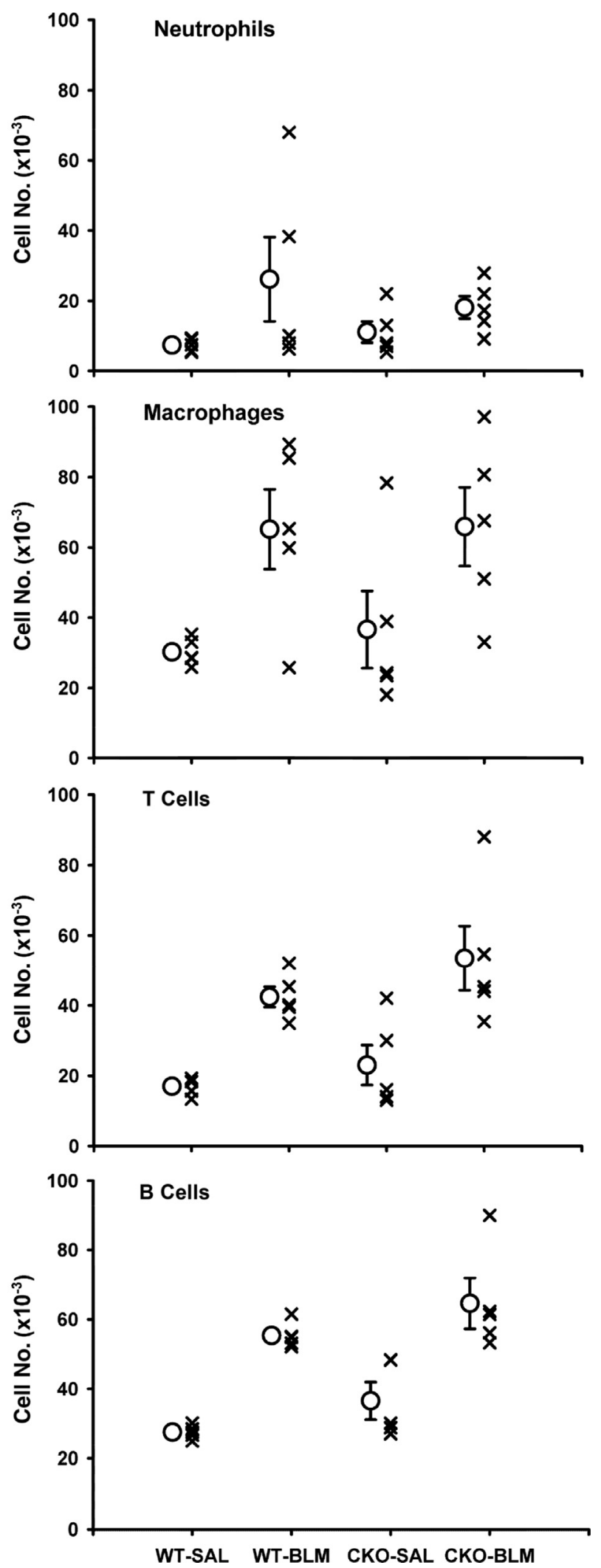

hemizygous for the Coll $\alpha 2-C r e-E R(T)$ allele with genotype $\left[\right.$ Notch $\left.1^{f l / f}, \operatorname{Coll} \alpha 2-\operatorname{Cre}-E R(T)^{+/ 0}\right]$. Genomic DNA from tail tissue samples of these mice was genotyped by PCR analysis. The presence of the WT, floxed Notchl and Cre-ER(T) genes was confirmed (Figure 1A). The appropriate mice were treated with tamoxifen, as described in Materials and Methods, to delete the Notchl gene in the mesenchymal compartment and are referred to as Notch1 CKO mice. Mice with genotype $\left[\mathrm{Notch}^{+/+}, \mathrm{Coll} \alpha 2-\mathrm{Cre}\right.$ $E R(T)^{+/ 0}$ ] and bearing the same $\operatorname{Cre}-E R(T)$ gene but with the WT Notchl gene were used as controls and were referred to as WT mice. The mesenchymal compartment deficiency of Notch1 expression was confirmed in lung fibroblasts isolated from Notch1 CKO mice (Figure 1B). Notch1 expression was intact in lung epithelial cells, neutrophils, macrophages, and $\mathrm{T}$ and $\mathrm{B}$ lymphocytes in Notch1 CKO mice (Supplemental Figure S1).

\section{Attenuated Fibrotic Response in Notch1 CKO Mice}

To evaluate the in vivo effect of mesenchymal Notch1 deficiency on pulmonary fibrosis, Notch1 CKO and control mice were treated with bleomycin, and the fibrotic response was evaluated. Lungs from saline-treated control and Notch1 CKO mice exhibited similar normal lung morphology (Figure 2A). Mesenchymal Notch1 deficiency for the duration of the experiment did not have a noticeable effect in the lung. However, in response to bleomycin treatment, the control (WT) mice showed the usual pulmonary fibrosis characterized by extensive distortion of alveolar architecture with thickened alveolar septae, inflammatory cell infiltration, increased number of fibroblasts, and extracellular matrix deposition (Figure 2A). In contrast, lungs of bleomycintreated Notch1 CKO mice revealed smaller areas affected by these fibrotic changes with a more patchy distribution (Figure 2A), suggesting a diminished fibrotic response when Notch1 was deficient in the mesenchymal compartment.

To quantitatively confirm this diminished pulmonary fibrosis in the Notch1 CKO mice, lung collagen deposition was biochemically analyzed for hydroxyproline content. Whole lung tissues from saline- or bleomycin-treated control and Notch1 CKO mice were obtained on day 21 after treatment. The results showed that bleomycin treatment in WT mice resulted in $>76 \%$ increase in lung hydroxyproline content relative to that in saline-treated WT controls (Figure 2B). In comparison, bleomycin-treated Notch1 CKO mice exhibited $<31 \%$ increase in lung hydroxyproline content relative to that in saline-treated Notch1 CKO mice. Thus, the increase in

Figure 3 Effect of mesenchymal Notch1 deficiency on bleomycininduced lung inflammation. Single-cell suspensions were prepared from the lungs of tamoxifen-treated Notch1 conditional knockout (CKO) and control [wild-type (WT)] mice receiving endotracheal injection of either bleomycin (BLM) or saline (SAL), as indicated. The samples were analyzed for the indicated inflammatory and immune cells by flow cytometry in 1 million of lung cells, and results are expressed as individual cell counts shown as Xs. Data are given as means \pm SEM (circles). $N=5$. 
lung hydroxyproline content caused by bleomycin treatment decreased by $>45 \%$ in Notch1 CKO mice relative to the increase seen in WT mice. Thus, Notch1 expression in the mesenchymal compartment played an important role in bleomycin-induced pulmonary fibrosis. The importance of Notch1 in this animal model has been suggested by activation of Hes1. ${ }^{21,51}$ This was confirmed by bleomycin-induced upregulation of lung Notch1 expression and that of its ligand Jagged1, with peak expression occurring on approximately day 7 after treatment in WT mice (Figure 2C). The decline in Notch1 expression at later time points suggested its importance primarily during the period of active fibrosis on days 7 to 14 after bleomycin treatment.

\section{Effect on Inflammation and Immune Response}

Because Notch1 expression in inflammatory and immune cells was not affected by the selective deficiency in the mesenchymal compartment, the observed diminished response to bleomycin-induced pulmonary fibrosis in CKO mice would not be because of a diminished inflammatory or immune response known to be activated in this animal model. $^{31,44,45}$ To evaluate this issue, whole lung single-cell suspensions from Notch1 CKO mice or WT controls were stained with fluorescent-labeled cell-specific antibodies and analyzed by flow cytometry to enumerate inflammatory and immune cells. The result showed that the number of neutrophils, macrophages, and $\mathrm{T}$ and $\mathrm{B}$ lymphocytes increased comparably in both WT and Notch CKO mice after bleomycin treatment (Figure 3). Thus, the importance of Notch1 appeared not to extend to the recruitment of inflammatory or immune cells in this animal model, and the noted reduction in fibrosis in CKO mice was not because of an antiinflammatory or immunosuppressive effect. However, the possibility of different cell recoveries between WT and CKO bleomycin-treated lungs masking an actual difference in inflammatory and immune response was not completely excluded.

\section{Effect on $\alpha$-SMA and Collagen Expression}

To further evaluate the effect of mesenchymal Notch1 deficiency on myofibroblast differentiation and fibrosis, lung tissue samples from control and CKO mice were evaluated for $\alpha$-SMA and type I collagen protein and mRNA levels. Consistent with the hydroxyproline analysis, Western blot analysis revealed significant bleomycin-induced increases in type I collagen protein in control, but not $\mathrm{CKO}$, mice (Figure 4A). Similarly, CKO mouse lung samples revealed an insignificant bleomycin-induced increase in $\alpha$-SMA protein in contrast to the robust response to bleomycin in control mice. Analysis of the corresponding lung mRNA levels revealed similar deficient responses to bleomycin in the CKO mouse samples relative to those from the control mouse samples (Figure 4B). Because de novo genesis of myofibroblasts in the bleomycin-induced fibrosis model corresponds to the induction
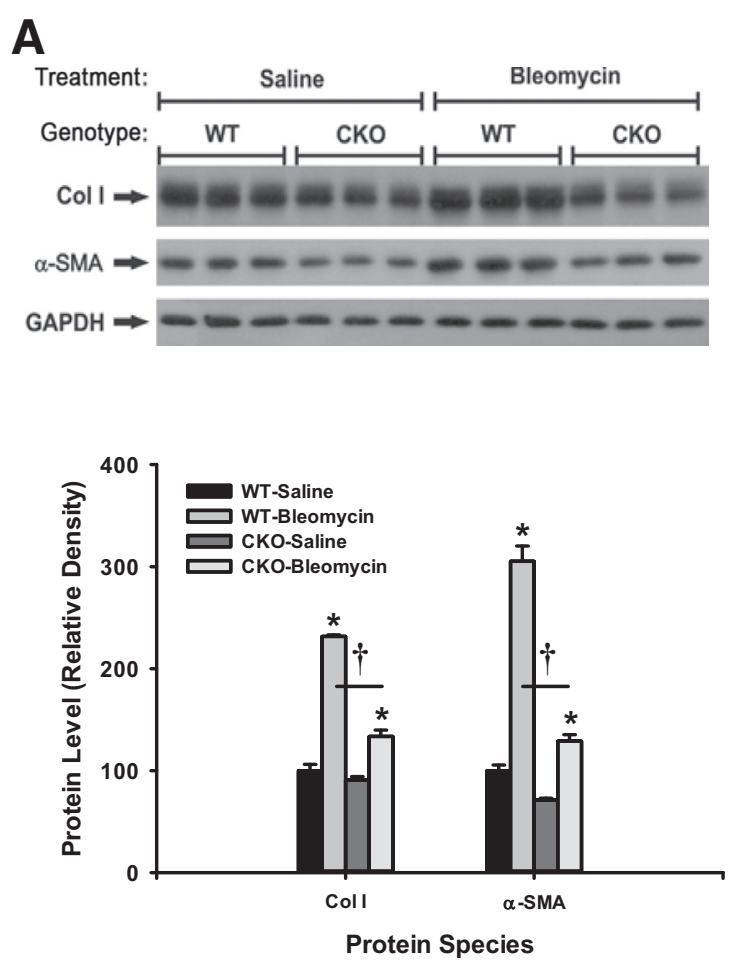

B

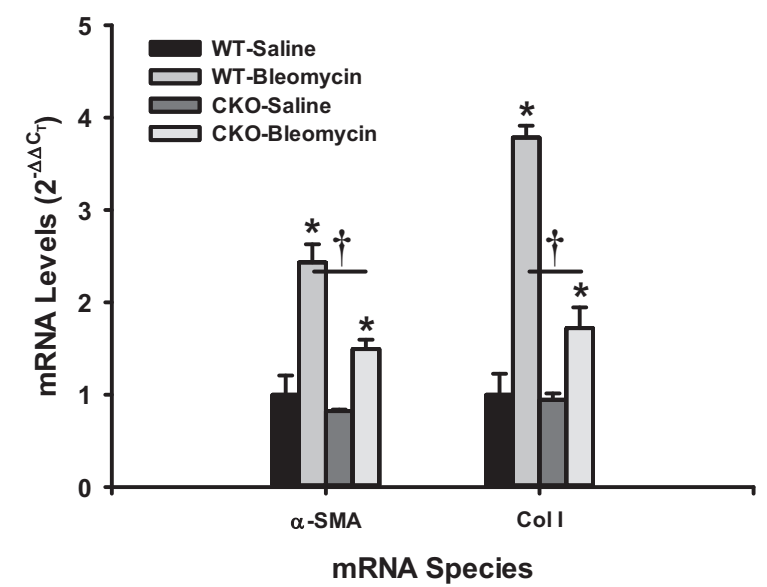

Figure 4 Effect of mesenchymal Notch1 deficiency on bleomycin-induced lung collagen expression and myofibroblast differentiation. Tamoxifen-treated control [wild-type (WT)] and Notch1 conditional knockout (CK0) mice were given endotracheal injections of either bleomycin or saline. Twenty-one days later, the lungs were harvested and analyzed for type I collagen (ColI), $\alpha$-smooth muscle actin ( $\alpha$-SMA), and Notch1 protein (A) and mRNA (B) by Western blot analysis and real-time PCR, respectively. A: Each lane represents a sample from a single animal; glyceraldehyde-3-phosphate dehydrogenase (GAPDH) was used as a loading control. The blots and their quantitation are shown. The bands were scanned, and the relative intensity of each protein was expressed as a percentage of the respective saline-treated wild-type control after normalization to the intensity of the respective GAPDH control band. B: $\alpha 1$ (I) Procollagen mRNA was detected by real-time PCR and results expressed as $2^{-\Delta \Delta C_{T}}$, with $18 \mathrm{~S}$ rRNA used as the reference and the level in saline-treated wild-type mice used as calibrator. Data were shown as means \pm SEM from triplicate samples (A and $\mathbf{B}$ ). ${ }^{*} P<0.05$, bleomycin-treated WT and CKO versus saline-treated WT and CKO, respectively; ${ }^{\dagger} P<0.05$ versus bleomycin-treated WT and bleomycintreated CKO lungs. 
of $\alpha$-SMA expression and myofibroblasts are the major type I collagen-producing cells, ${ }^{27,52,53}$ diminished induction of these two genes in CKO mice would be consistent with deficient myofibroblast differentiation in the absence of Notch1 in the mesenchymal compartment.

\section{Reduction of Myofibroblasts in Notch1 CKO Mice}

To further evaluate the importance of Notch1 in myofibroblast differentiation in bleomycin-induced pulmonary fibrosis, lung fibroblasts were isolated from Notch1 CKO and control (WT) mice after saline or bleomycin treatment. As expected, Notch 1 expression was intact in cells from WT mice with upregulation in those from bleomycin-treated WT mice, but was undetectable in the CKO mouse cell samples (Figure 5A). Both $\alpha$-SMA and type I collagen expression levels were increased in lung fibroblasts from bleomycin-treated WT mice at both protein (Figure 5A) and mRNA (Figure 5B) levels relative to cells from saline-treated WT mice. In contrast, the corresponding increases in cells from bleomycin-treated CKO mice were of lesser magnitude and significantly less at the mRNA level relative to that seen in cells from WT mice.

Immunofluorescence analysis (using anti- $\alpha$-SMA antibodies) of lung fibroblasts from CKO mice revealed fewer $\alpha$ SMA-positive cells and much weaker expression of $\alpha$-SMA relative to cells from WT mice (Figure 6A). Moreover, the morphology of most of the CKO cells was consistent with less differentiated fibroblasts in contrast to the more spread out morphology with distinct $\alpha$-SMA fiber formation of the WT cells. Enumeration of the lung $\alpha$-SMA-positive cells by flow cytometry confirmed that they were increased by more than threefold in bleomycin-treated WT mice, which was significantly less (approximately twofold) in CKO mice (Figure 6B). Thus, Notch1 deficiency in the mesenchymal compartment resulted in significantly reduced myofibroblast differentiation contributing to the noted reduction in pulmonary fibrosis.

\section{Discussion}

Genesis of the myofibroblast and its persistence are key factors in chronic fibroproliferative diseases with significant tissue fibrosis. ${ }^{27,52,53}$ In the lung, Notch1 is implicated in pulmonary fibrosis, presumably through its regulation of lung cytokine expression, myofibroblast differentiation, and collagen deposition. ${ }^{21}$ Thus, Notch1 appears to regulate multiple cellular functions in various cell types with differing target genes. ${ }^{1,2,6,12,23,54}$ Distinguishing the various important roles and possible mechanisms of this molecule in different cell types is important to determine its precise role(s) in development and disease.

Notch1 is known to regulate the inflammatory response, including the acute phase response, which may also affect pulmonary fibrosis via indirect mechanisms in contrast to directly affecting matrix-producing mesenchymal cells. ${ }^{2,11,21,55-57}$
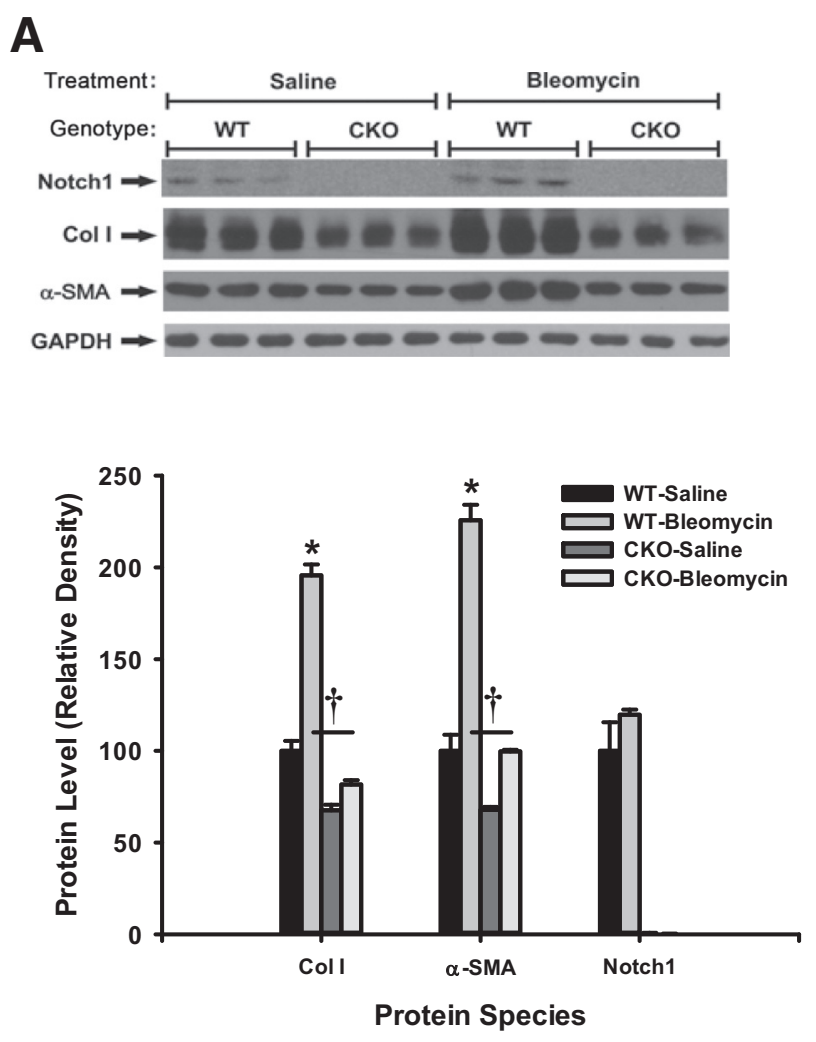

B

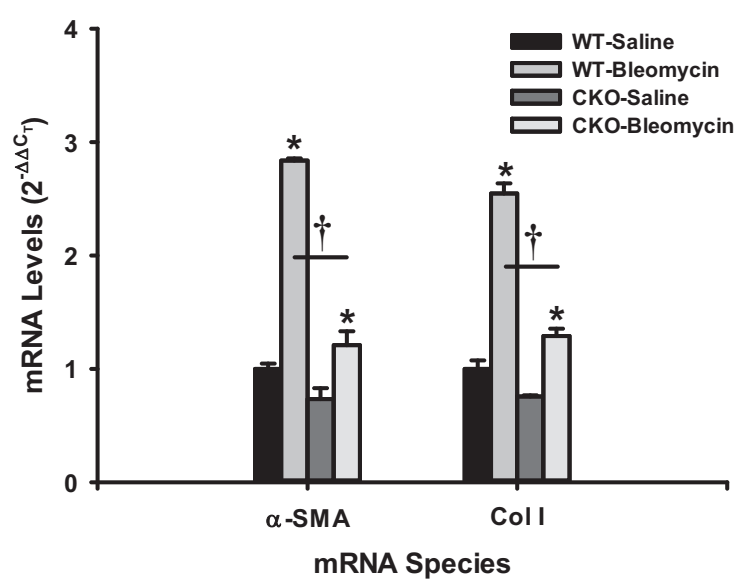

Figure 5 Effect of Notch1 deficiency in mesenchymal compartment on lung fibroblasts. Wild-type (WT) and Notch1 conditional knockout (CKO) mice were injected endotracheally with saline or bleomycin, as indicated. A: Fourteen days after injection, lung fibroblasts from these mice were isolated and analyzed for Notch1, collagen I (ColI), and $\alpha$ smooth muscle actin ( $\alpha$-SMA) proteins by Western blot analysis. The bands were scanned and the relative intensity of each protein was expressed as a percentage of the respective saline-treated WT control after normalization to the intensity of the respective glyceraldehyde-3phosphate dehydrogenase (GAPDH). Absence of Notch1 expression was confirmed in cells from saline- or bleomycin-treated CKO mice; its expression, as well as that for ColI and $\alpha$-SMA, was elevated in bleomycin-treated WT cells but not in CKO cells. B: Analysis of ColI and $\alpha$ SMA mRNAs by real-time PCR also confirmed significantly higher levels of expression in cells from bleomycin-treated WT or CKO mice. ${ }^{*} P<0.05$ versus WT and CKO saline-treated lung; ${ }^{\dagger} P<0.05$ versus WT and CKO bleomycin-treated lungs. 

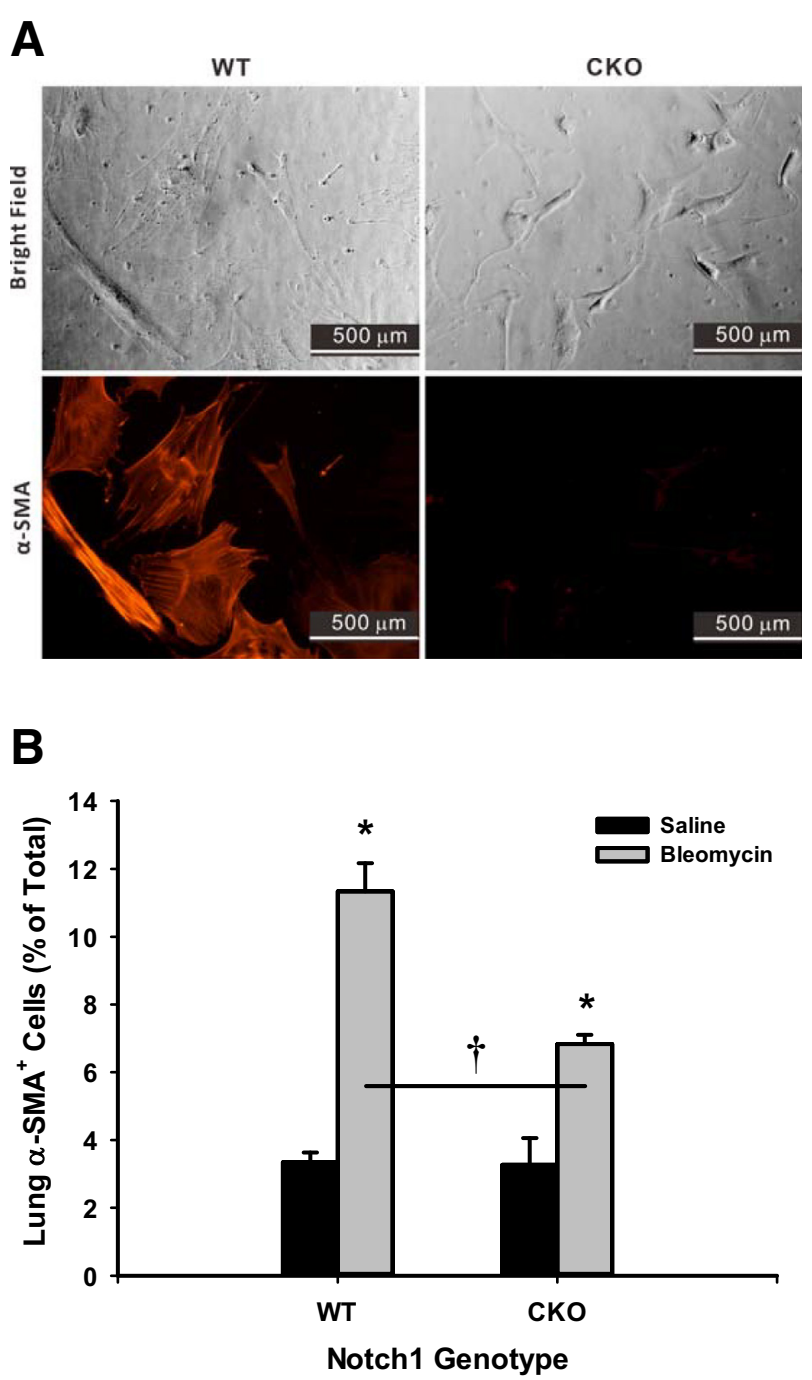

Figure 6 Notch1 regulates lung myofibroblast differentiation. A: Lung fibroblasts isolated from wild-type (WT) control or Notch1 conditional knockout (CKO) mice [bright-field images (top panels)] were immunostained with Cy3-conjugated anti- $\alpha$-smooth muscle actin ( $\alpha$-SMA) antibody (bottom panels). B: Mice of the indicated strains were treated with saline or bleomycin after tamoxifen injection. Single-cell suspensions were obtained from lung tissue by enzymatic digestion and analyzed for $\alpha$-SMA-positive cells by flow cytometry. The results were expressed as the percentage of $\alpha$-SMA-positive cells in the total lung cell suspension. Data were shown as means \pm SEM from triplicate samples (B). ${ }^{*} P<0.05$ versus WT and CKO saline-treated controls; ${ }^{\dagger} P<0.05$ versus WT and CKO bleomycin-treated lungs. Original magnification, $\times 200$ (A).

Notch1 is also involved in regulation of other lung cell types, which may also have an impact on myofibroblast differentiation and fibrosis. ${ }^{9,12,21,23,55}$ Indeed, pulmonary fibrosis is diminished in mice with impaired Notch signaling because of diminished fucosylation of Notch proteins as a result of deficiency in GDP-4-keto-6-deoxymannose-3,5-epimerase-4reductase. ${ }^{21}$ Because Notch1 in all cell compartments is inactivated by this approach, it is difficult to discriminate the effects of Notch1 in different cells and tissues. Thus, our aim was to evaluate the specific importance of Notch1 in the mesenchymal compartment in the induction of myofibroblast differentiation in vivo, specifically in the pathogenesis of pulmonary fibrosis, and to distinguish it from its potential effects on other compartments, such as the inflammatory and immune compartment. This was achieved by using transgenic mice expressing the Cre-ER(T) recombinase only in collagen I-expressing cells (with the aid of the collagen I promoter enhancer) in conjunction with the homozygous floxed Notchl allele.

When the Cre-ER(T) recombinase was activated by tamoxifen, Notch1 expression was selectively absent only in collagen I-expressing cells, whereas epithelial, inflammatory, and immune cells were unaffected. When bleomycin-induced pulmonary fibrosis was induced in these mice, mesenchymalspecific loss of Notch1 resulted in significantly reduced lung fibrosis on the basis of both histopathological evaluation and quantitative biochemical analysis. Results of additional analysis of $\alpha$-SMA and collagen I gene expression and enumeration of lung $\alpha$-SMA-positive cells were consistent with reduced myofibroblast differentiation in the lungs of Notch1 CKO mice on bleomycin challenge. Because myofibroblasts are the major source of fibrogenic cytokine and extracellular matrix, ${ }^{27,52,53}$ this reduction in myofibroblast accumulation in the Notch1 CKO mice would be expected to contribute to the noted significant reduction in fibrosis. This result is also consistent with previous studies suggesting the importance of Notch1 in fibrosis and in myofibroblast differentiation in vitro. ${ }^{21}$ Of further interest is the lack of significant effect of mesenchymal Notch1 deficiency on the inflammatory and immune cell recruitment in response to bleomycin treatment, thus affirming the selective significance of Notch1 in the mesenchymal compartment vis-à-vis myofibroblast differentiation and fibrosis. Similarly, the findings implied that despite intact Notch1 in the epithelial and endothelial compartments, its absence in the mesenchymal compartment significantly impaired fibrosis and genesis of the myofibroblast.

However, the diminished fibrosis in the absence of Notch1 in mesenchymal cells was incomplete and the lung histopathology still showed significant fibrosis, albeit affecting smaller areas of lung tissue. This would indicate that Notch1 could also play additional roles in nonmesenchymal cells and/or the loss of Notch1 expression in the mesenchymal compartment was incomplete. In the former case, the additional contribution of Notch1 in other cellular compartments is a consideration given that Notch1 is a known regulator of epithelial regeneration, ${ }^{38}$ T-cell differentiation, and pulmonary inflammation. ${ }^{37}$

In the current study, recruitment of inflammatory and immune cells in bleomycin-treated mice was not significantly affected by Notch1 deficiency in the mesenchymal compartment, suggesting unlikely contribution from the immune and inflammatory compartment with respect to the role of Notch1. However, this was not completely excluded because of some uncertainty with respect to potential differences in cell recovery from lung tissue with differing levels of fibrosis. In the case of the epithelial compartment, recent evidence suggests that persistent Notch signaling may be responsible for the development of honeycombing in 
end-stage pulmonary fibrosis. ${ }^{58}$ Another possibility for incomplete inhibition of fibrosis in Notch1 CKO mice is that regulation of myofibroblast differentiation is complex and can be induced by alternative pathways, such as via transforming growth factor- $\beta /$ Smad and Hedgehog. ${ }^{29,44,59}$ Finally, although Notch1 expression was not detectable in lung fibroblasts in CKO mice, expression lower than the limits of detection could not be excluded, which could account for the $<100 \%$ suppression of fibrosis noted.

In conclusion, our findings demonstrate the specific importance of Notch1 expression in the mesenchymal compartment in pulmonary fibrosis. The mechanism was at least in part because of significant impairment of myofibroblast differentiation. Although the results confirmed the importance of mesenchymal Notch1 on myofibroblast differentiation in vivo, additional and/or alternate mechanisms cannot be excluded because of incomplete impairment of fibrosis. Additional future studies are warranted to more precisely examine this contribution by the nonmesenchymal compartments.

\section{Acknowledgments}

We thank Kelli Rule for breeding the mice and Lisa R. Riggs for technical assistance.

\section{Supplemental Data}

Supplemental material for this article can be found at http://dx.doi.org/10.1016/j.ajpath.2015.07.014.

\section{References}

1. Artavanis-Tsakonas S, Rand MD, Lake RJ: Notch signaling: cell fate control and signal integration in development. Science 1999, 284: 770-776

2. Radtke F, Fasnacht N, Macdonald HR: Notch signaling in the immune system. Immunity 2010, 32:14-27

3. Aster JC, Blacklow SC, Pear WS: Notch signalling in T-cell lymphoblastic leukaemia/lymphoma and other haematological malignancies. J Pathol 2011, 223:262-273

4. Kim TH, Shivdasani RA: Notch signaling in stomach epithelial stem cell homeostasis. J Exp Med 2011, 208:677-688

5. Miyamoto S, Rosenberg DW: Role of Notch signaling in colon homeostasis and carcinogenesis. Cancer Sci 2011, 102:1938-1942

6. Sethi N, Kang Y: Notch signalling in cancer progression and bone metastasis. Br J Cancer 2011, 105:1805-1810

7. van der Fits L, Qin Y, Out-Luiting JJ, Vermeer KG, Whittaker S, van Es JH, Tensen CP, Vermeer MH: NOTCH1 signaling as a therapeutic target in Sezary syndrome. J Invest Dermatol 2012, 132: $2810-2817$

8. VanDussen KL, Carulli AJ, Keeley TM, Patel SR, Puthoff BJ, Magness ST, Tran IT, Maillard I, Siebel C, Kolterud A, Grosse AS, Gumucio DL, Ernst SA, Tsai YH, Dempsey PJ, Samuelson LC: Notch signaling modulates proliferation and differentiation of intestinal crypt base columnar stem cells. Development 2012, 139: 488-497

9. Noah TK, Shroyer NF: Notch in the intestine: regulation of homeostasis and pathogenesis. Annu Rev Physiol 2013, 75:263-288
10. Xu K, Moghal N, Egan SE: Notch signaling in lung development and disease. Adv Exp Med Biol 2012, 727:89-98

11. Seifert T, Bauer J, Weissert R, Fazekas F, Storch MK: Notch1 and its ligand Jagged 1 are present in remyelination in a T-cell- and antibodymediated model of inflammatory demyelination. Acta Neuropathol 2007, 113:195-203

12. Wael H, Yoshida R, Kudoh S, Hasegawa K, Niimori-Kita K, Ito T: Notch1 signaling controls cell proliferation, apoptosis and differentiation in lung carcinoma. Lung Cancer 2014, 85:131-140

13. Gordon WR, Arnett KL, Blacklow SC: The molecular logic of Notch signaling: a structural and biochemical perspective. J Cell Sci 2008 , 121:3109-3119

14. Shao L, Luo Y, Moloney DJ, Haltiwanger R: O-glycosylation of EGF repeats: identification and initial characterization of a UDP-glucose: protein O-glucosyltransferase. Glycobiology 2002, 12:763-770

15. Ma B, Simala-Grant JL, Taylor DE: Fucosylation in prokaryotes and eukaryotes. Glycobiology 2006, 16:158R-184R

16. Moloney DJ, Panin VM, Johnston SH, Chen J, Shao L, Wilson R, Wang Y, Stanley P, Irvine KD, Haltiwanger RS, Vogt TF: Fringe is a glycosyltransferase that modifies Notch. Nature 2000, 406:369-375

17. LeBon L, Lee TV, Sprinzak D, Jafar-Nejad H, Elowitz MB: Fringe proteins modulate Notch-ligand cis and trans interactions to specify signaling states. Elife 2014, 3:e02950

18. Irvine KD: Fringe, Notch, and making developmental boundaries. Curr Opin Genet Dev 1999, 9:434-44

19. Zhou L, Li LW, Yan Q, Petryniak B, Man Y, Su C, Shim J, Chervin S, Lowe JB: Notch-dependent control of myelopoiesis is regulated by fucosylation. Blood 2008, 112:308-319

20. Becker DJ, Lowe JB: Fucose: biosynthesis and biological function in mammals. Glycobiology 2003, 13:41R-53R

21. Liu T, Hu B, Choi YY, Chung M, Ullenbruch M, Yu H, Lowe JB Phan SH: Notch1 signaling in FIZZ1 induction of myofibroblast differentiation. Am J Pathol 2009, 174:1745-1755

22. Syed F, Bayat A: Notch signaling pathway in keloid disease: enhanced fibroblast activity in a Jagged-1 peptide-dependent manner in lesional vs. extralesional fibroblasts. Wound Repair Regen 2012, 20:688-706

23. Hu M, Ou-Yang HF, Wu CG, Qu SY, Xu XT, Wang P: Notch signaling regulates collalpha1 and collalpha2 expression in airway fibroblasts. Exp Biol Med (Maywood) 2014, 239:1589-1596

24. Zhang K, Zhang YQ, Ai WB, Hu QT, Zhang QJ, Wan LY, Wang XL, Liu CB, Wu JF: Hes1, an important gene for activation of hepatic stellate cells, is regulated by Notch1 and TGF-beta/BMP signaling. World J Gastroenterol 2015, 21:878-887

25. Gharaee-Kermani M, Hu B, Thannickal VJ, Phan SH, Gyetko MR: Current and emerging drugs for idiopathic pulmonary fibrosis. Expert Opin Emerg Drugs 2007, 12:627-646

26. Hu B, Phan SH: Myofibroblasts. Curr Opin Rheumatol 2013, 25:71-77

27. Zhang K, Rekhter MD, Gordon D, Phan SH: Myofibroblasts and their role in lung collagen gene expression during pulmonary fibrosis: a combined immunohistochemical and in situ hybridization study. Am J Pathol 1994, 145:114-125

28. Zhang K, Gharaee-Kermani M, McGarry B, Phan SH: In situ hybridization analysis of rat lung alpha 1(I) and alpha 2(I) collagen gene expression in pulmonary fibrosis induced by endotracheal bleomycin injection. Lab Invest 1994, 70:192-202

29. Hinz B, Phan SH, Thannickal VJ, Prunotto M, Desmouliere A, Varga J, De Wever O, Mareel M, Gabbiani G: Recent developments in myofibroblast biology: paradigms for connective tissue remodeling. Am J Pathol 2012, 180:1340-1355

30. Phan SH: Biology of fibroblasts and myofibroblasts. Proc Am Thorac Soc 2008, 5:334-337

31. Gharaee-Kermani M, Phan SH: Molecular mechanisms of and possible treatment strategies for idiopathic pulmonary fibrosis. Curr Pharm Des 2005, 11:3943-3971

32. Xu K, Nieuwenhuis E, Cohen BL, Wang W, Canty AJ, Danska JS, Coultas L, Rossant J, Wu MY, Piscione TD, Nagy A, Gossler A, Hicks GG, Hui CC, Henkelman RM, Yu LX, Sled JG, Gridley T, 
Egan SE: Lunatic Fringe-mediated Notch signaling is required for lung alveogenesis. Am J Physiol Lung Cell Mol Physiol 2010, 298: L45-L56

33. Zhang S, Loch AJ, Radtke F, Egan SE, Xu K: Jagged1 is the major regulator of Notch-dependent cell fate in proximal airways. Dev Dyn 2013, 242:678-686

34. Mori M, Mahoney JE, Stupnikov MR, Paez-Cortez JR, Szymaniak AD, Varelas X, Herrick DB, Schwob J, Zhang H, Cardoso WV: Notch3-Jagged signaling controls the pool of undifferentiated airway progenitors. Development 2015, 142:258-267

35. Xing Y, Li A, Borok Z, Li C, Minoo P: NOTCH1 is required for regeneration of Clara cells during repair of airway injury. Stem Cells 2012, 30:946-955

36. Katano T, Ootani A, Mizoshita T, Tanida S, Tsukamoto H, Ozeki K, Kataoka H, Joh T: Gastric mesenchymal myofibroblasts maintain stem cell activity and proliferation of murine gastric epithelium in vitro. Am J Pathol 2015, 185:798-807

37. Zhou M, Cui ZL, Guo XJ, Ren LP, Yang M, Fan ZW, Han RC, Xu WG: Blockade of Notch signalling by gamma-secretase inhibitor in lung $\mathrm{T}$ cells of asthmatic mice affects $\mathrm{T}$ cell differentiation and pulmonary inflammation. Inflammation 2015, 38:1281-1288

38. Whitsett JA, Kalinichenko VV: Notch and basal cells take center stage during airway epithelial regeneration. Cell Stem Cell 2011, 8: 597-598

39. Luzina IG, Todd NW, Sundararajan S, Atamas SP: The cytokines of pulmonary fibrosis: much learned, much more to learn. Cytokine 2015, 74:88-100

40. Todd NW, Luzina IG, Atamas SP: Molecular and cellular mechanisms of pulmonary fibrosis. Fibrogenesis Tissue Repair 2012, 5:11

41. Feil R, Brocard J, Mascrez B, LeMeur M, Metzger D, Chambon P: Ligand-activated site-specific recombination in mice. Proc Natl Acad Sci U S A 1996, 93:10887-10890

42. Bou-Gharios G, Garrett LA, Rossert J, Niederreither K, Eberspaecher H, Smith C, Black C, Crombrugghe B: A potent farupstream enhancer in the mouse pro alpha 2(I) collagen gene regulates expression of reporter genes in transgenic mice. J Cell Biol 1996, 134:1333-1344

43. Hayashi S, McMahon AP: Efficient recombination in diverse tissues by a tamoxifen-inducible form of Cre: a tool for temporally regulated gene activation/inactivation in the mouse. Dev Biol 2002, 244:305-318

44. Hu B, Liu J, Wu Z, Liu T, Ullenbruch MR, Ding L, Henke CA, Bitterman PB, Phan SH: Reemergence of hedgehog mediates epithelial-mesenchymal crosstalk in pulmonary fibrosis. Am J Respir Cell Mol Biol 2015, 52:418-428

45. Hu B, Wu Z, Nakashima T, Phan SH: Mesenchymal-specific deletion of C/EBPbeta suppresses pulmonary fibrosis. Am J Pathol 2012, 180: $2257-2267$

46. Zheng B, Zhang Z, Black CM, de Crombrugghe B, Denton CP: Ligand-dependent genetic recombination in fibroblasts: a potentially powerful technique for investigating gene function in fibrosis. Am J Pathol 2002, 160:1609-1617
47. Zheng J, Watanabe H, Wines-Samuelson M, Zhao H, Gridley T, Kopan R, Shen J: Conditional deletion of Notch1 and Notch2 genes in excitatory neurons of postnatal forebrain does not cause neurodegeneration or reduction of Notch mRNAs and proteins. J Biol Chem 2012, 287:20356-20368

48. Yang X, Klein R, Tian X, Cheng HT, Kopan R, Shen J: Notch activation induces apoptosis in neural progenitor cells through a p53dependent pathway. Dev Biol 2004, 269:81-94

49. Hu B, Wu Z, Phan SH: Smad3 mediates transforming growth factorbeta-induced alpha-smooth muscle actin expression. Am J Respir Cell Mol Biol 2003, 29:397-404

50. Livak KJ, Schmittgen TD: Analysis of relative gene expression data using real-time quantitative PCR and the 2(-Delta Delta C(T)) Method. Methods 2001, 25:402-408

51. Bielesz B, Sirin Y, Si H, Niranjan T, Gruenwald A, Ahn S, Kato H, Pullman J, Gessler M, Haase VH, Susztak K: Epithelial Notch signaling regulates interstitial fibrosis development in the kidneys of mice and humans. J Clin Invest 2010, 120:4040-4054

52. Zhang HY, Gharaee-Kermani M, Zhang K, Karmiol S, Phan SH: Lung fibroblast alpha-smooth muscle actin expression and contractile phenotype in bleomycin-induced pulmonary fibrosis. Am J Pathol 1996, 148:527-537

53. Phan SH: Genesis of the myofibroblast in lung injury and fibrosis. Proc Am Thorac Soc 2012, 9:148-152

54. Gao J, Dong Y, Zhang B, Xiong Y, Xu W, Cheng Y, Dai M, Yu Z, $\mathrm{Xu} \mathrm{H}$, Zheng G: Notch1 activation contributes to tumor cell growth and proliferation in human hepatocellular carcinoma HepG2 and SMMC7721 cells. Int J Oncol 2012, 41:1773-1781

55. Outtz HH, Wu JK, Wang X, Kitajewski J: Notch1 deficiency results in decreased inflammation during wound healing and regulates vascular endothelial growth factor receptor-1 and inflammatory cytokine expression in macrophages. J Immunol 2010, 185: 4363-4373

56. Monsalve E, Ruiz-Garcia A, Baladron V, Ruiz-Hidalgo MJ, SanchezSolana B, Rivero S, Garcia-Ramirez JJ, Rubio A, Laborda J, DiazGuerra MJ: Notch1 upregulates LPS-induced macrophage activation by increasing NF-kappaB activity. Eur J Immunol 2009, 39: $2556-2570$

57. Zhang Q, Wang C, Liu Z, Liu X, Han C, Cao X, Li N: Notch signal suppresses Toll-like receptor-triggered inflammatory responses in macrophages by inhibiting extracellular signal-regulated kinase 1/2mediated nuclear factor kappaB activation. J Biol Chem 2012, 287: $6208-6217$

58. Vaughan AE, Brumwell AN, Xi Y, Gotts JE, Brownfield DG, Treutlein B, Tan K, Tan V, Liu FC, Looney MR, Matthay MA, Rock JR, Chapman HA: Lineage-negative progenitors mobilize to regenerate lung epithelium after major injury. Nature 2015, 517: $621-625$

59. Hinz B, Phan SH, Thannickal VJ, Galli A, Bochaton-Piallat ML, Gabbiani G: The myofibroblast: one function, multiple origins. Am J Pathol 2007, 170:1807-1816 